\title{
IST: suas principais complicações durante a gravidez
}

\author{
STIs: its main complications during pregnancy \\ ITS: sus principales complicaciones durante el embarazo
}

Recebido: 02/11/2021 | Revisado: 07/11/2021 | Aceito: 08/12/2021 | Publicado: 15/12/2021

\author{
Euriany Maria Souza da Silva \\ ORCID: https://orcid.org/0000-0003-3962-6919 \\ Centro Universitário da Amazônia, Brasil \\ E-mail: eurianysouza96@gmail.com \\ Sandy Sampaio Cardoso \\ ORCID: https://orcid.org/0000-0001-8623-2934 \\ Centro Universitário da Amazônia, Brasil \\ E-mail: sandysampaio16.hada@gmail.com \\ Ingrid da Silva Leite \\ ORCID: https://orcid.org/0000-0002-2627-2675 \\ Centro Universitário da Amazônia, Brasil \\ E-mail: ingridleitestm@gmail.com
}

\begin{abstract}
Resumo
Introdução: As infecções sexualmente transmissíveis (IST) é assunto a ser discutido na saúde pública, ela encontra-se entre as principais causas de procura por assistência no mundo, elas afetam ambos os sexos, sendo explicito na mortalidade materna e infantil. Objetivo: Esclarecer as principais IST mais comuns durante o período da gravidez, e quais são as complicações mais recorrentes materno-infantil. Metodologia: Trata-se de uma revisão sistemática da literatura de caráter qualitativo, relacionado a complicações por IST durante gravidez. Para identificar estudos, realizouse buscas nas bases de dados eletrônicas, Latino Americana (LILACS), US National Library of Medicine (PUBMED), Cumulative Index to Nursing \& Allied Health Literature (CINAHL), SciVerse Scopus (SCOPUS), Cochrane Library (COCHRANE) e Google Scholar. Com a seguinte estratégia de busca por meio do cruzamento dos descritores utilizando a lógica dos recursos booleanos no campo de busca, no período de 2016 à 2021. Resultado: As gestantes que não fazem uso de preservativos são as que correm mais riscos de contrair alguma IST e o rastreamento preconcepção e pré-natal na mãe, ou neonatal na criança, que estão disponíveis, é fundamental para o diagnóstico e tratamento precoces. Conclusão: O Enfermeiro diante dessas situações, é um principal agente na atenção básica através do pré-natal, onde é realizado o diagnóstico, ofertado o tratamento e feito o acolhimento dessa gestante.
\end{abstract}

Palavras-chave: Cuidado pré natal; Infecções sexualmente transmissíveis; Saúde materno-infantil.

\begin{abstract}
Introduction: Sexually transmitted infections (STIs) are a matter to be discussed in public health, they are among the main causes of seeking care in the world, they affect both sexes, being explicit in maternal and child mortality. Objective: To clarify the main most common STIs during the pregnancy period, and which are the most recurrent maternal-infant complications. Methodology: This is a systematic review of the literature of a qualitative nature, related to complications due to STIs during pregnancy. To identify studies, searches were performed in electronic databases, Latin American (LILACS), US National Library of Medicine (PUBMED), Cumulative Index to Nursing \& Allied Health Literature (CINAHL), SciVerse Scopus (SCOPUS), Cochrane Library (COCHRANE) and Google Scholar. With the following search strategy through the crossing of descriptors using the logic of Boolean resources in the search field, in the period from 2016 to 2021. Result: Pregnant women who do not use condoms are at the highest risk of contracting an STI and preconception and prenatal screening in the mother, or neonatal screening in the child, which are available, is essential for early diagnosis and treatment. Conclusion: The nurse in these situations is a main agent in primary care through prenatal care, where the diagnosis is performed, the treatment offered and the reception of this pregnant woman performed.
\end{abstract}

Keywords: Prenatal care; Sexually transmitted infections; Maternal-child nursing.

\section{Resumen}

Introducción: Las infecciones de transmisión sexual (ITS) son un tema a discutir en salud pública, se encuentran entre las principales causas de búsqueda de atención en el mundo, afectan a ambos sexos, siendo explícitos en la mortalidad materna e infantil. Objetivo: aclarar las principales ITS más frecuentes durante el período de gestación y cuáles son las complicaciones materno-infantiles más recurrentes. Metodología: Se trata de una revisión sistemática de la literatura de carácter cualitativo, relacionada con las complicaciones por ITS durante el embarazo. Para identificar los estudios, se realizaron búsquedas en bases de datos electrónicas, América Latina (LILACS), Biblioteca Nacional de Medicina de EE. UU. (PUBMED), Índice acumulativo de literatura de enfermería y salud afín (CINAHL), SciVerse Scopus 
(SCOPUS), Biblioteca Cochrane (COCHRANE) y Google Académico. Con la siguiente estrategia de búsqueda mediante el cruce de descriptores utilizando la lógica de los recursos booleanos en el campo de búsqueda, en el período de 2016 a 2021. Resultado: Las mujeres embarazadas que no usan condón tienen mayor riesgo de contraer una ITS y preconcepción y El cribado prenatal en la madre o el cribado neonatal en el niño, que están disponibles, son esenciales para el diagnóstico y el tratamiento precoces. Conclusión: La enfermera en estas situaciones es un agente principal en la atención primaria a través de la atención prenatal, donde se realiza el diagnóstico, el tratamiento ofrecido y la recepción de esta gestante.

Palabras clave: Atención Prenatal; Infecciones de transmisión sexual; Enfermería materno infantil.

\section{Introdução}

A região Norte do Brasil destaca-se no cenário nacional como a que detém maior crescimento nos coeficientes de detecção de HIV em gestantes nos últimos dez anos. Dentro desse panorama epidemiológico, o estado do Pará apresentou a quarta maior taxa do país de detecção de HIV em gestantes, registrando índice de 3,4 casos/mil nascidos vivos em 2017 (Trindade et al. 2020).

Essas informações foram coletadas através de pesquisas e produção científica sobre Infecções Sexualmente Transmissíveis (IST) que são relevantes, pois tais infecções têm sido prevalentes na população em geral e em gestantes e nestas podem acarretar complicações obstétricas e neonatais aumentando a morbimortalidade materno-infantil.

Em decorrência disso, há uma necessidade de que haja um maior o aprofundamento científico, no sentido de elevar as chances de contribuição para a redução dos índices de contaminação e consequentemente para a melhoria na qualidade de vida das pessoas (Silva et al. 2020).

De acordo com Melo et al. (2018), o vírus B da hepatite (HBV), que pode ser transmitido por contato parenteral, perinatal ou sexual, é uma doença infecciosa grave do fígado. A infecção por vírus B da hepatite - HBV durante a gravidez está associada a um alto risco de complicações maternas, alta taxa de transmissão vertical causando hepatite fetal e neonatal e maior mortalidade materna.

O mesmo autor, ainda ressalta que a sífilis é uma (IST) curável e exclusiva do ser humano, e é transmitida por contato sexual, transfusão de sangue e via transmissão vertical, é um grave problema de saúde, principalmente na África Subsaariana. A sífilis em mulheres grávidas causa infecção congênita grave, que resulta em morte fetal / neonatal (50\%), prematuridade (25\%) e sequelas importantes de longo prazo em crianças sobreviventes (20\%).

Conforme Joseph et al (2011), o herpes genital na gravidez causa morbidade materna significativa, com um número crescente de infecções devido à transmissão oral-labial. Perto do parto, as infecções primárias com herpes-vírus simples HSV-1 ou HSV-2 representam o maior risco de infecção por herpes neonatal, que é uma infecção rara, mas potencialmente devastadora para recém-nascidos.

Para Violari et al. (2008), o vírus da imunodeficiência humana HIV, como as outras ISTs, tem um efeito severo em mulheres grávidas. Estudos têm mostrado resultados adversos da gravidez em mulheres infectadas pelo HIV. A orientação nos atendimentos de consulta de pré-natal é de suma importância, pois muitas mães são de primeira viagem e não tem o conhecimento adequado para lidar com situações como essas, onde muitas vezes por falta de orientação acabam colocando sua vida e a do feto em risco. Justifica-se a pesquisa pela ocorrência de muitos casos de Infecções Sexualmente Transmissíveis (IST), em gestantes na qual se coloca em risco a vida da mãe e do bebê, podendo leva-los a morbimortalidade materno-infantil.

Com base nesse contexto, é de fundamental importância orientar e contribuir com a redução da morbimortalidade materno-infantil, possibilitando mostrar a realidade vivida pelas grávidas e suas responsabilidades em proteger-se cuidando de si para se ter bebês saudáveis sem risco a vida. Sabe-se que tratar desse assunto é delicado, pois há mães que pouco se interessam em ter um acompanhamento e diagnosticar as fazes de desenvolvimento do feto, outras frequentam o pré natal, mais não levam 
a sério o diagnostico apresentado pelos médicos, isso porque são mães do primeiro filho e acreditam que só o fato da barriga está crescendo tudo está normal sem complicações.

Diante do cenário exposto, este estudo tem como objetivo esclarecer as principais IST's mais comuns durante o período da gravidez, e quais são as complicações mais recorrentes materno-infantil. Com essa holística, justifica-se a necessidade de especialistas de diversas áreas de saúde, e em especial aos enfermeiros que trabalham diretamente no acompanhamento e monitoramento das mães grávida e materno infantil ter um olhar sensível a essa rotina, identificar os sintomas e direcionar ao atendimento médico que vai examinar para detectar qual IST a mãe possui.

O médico por sua vez, vai diagnóstica, orientar a melhor forma do medicamento a ser ingerido pela enferma, que não possam interferir na gestação e saúde do bebê, e possibilitar o tratamento, acompanhamento adequado a cada mãe de acordo com suas necessidades e cuidado com a vida. Diante dessa informação, percebe-se que o enfermeiro desempenha uma função axial que é identificar alguma anormalidade durante a gravidez e suas principais complicações e direcioná-las ao tratamento médico quando assim for necessário, além disso, ele realiza durante seu atendimento orientação a prevenção e cuidados com as ISTs (Andrade, 2021).

Portanto, exames de rotina e o pré-natal é essencial para o acompanhamento das gestantes, pois o diagnóstico precoce de alguma infecção sexualmente transmissível (IST) é importante para que ocorra o tratamento adequado o mais rápido possível, eliminando correlativamente o grau de risco de vida do feto e da mãe. Diante disso, o objetivo deste estudo é esclarecer as principais IST mais comuns durante o período da gravidez, e quais são as complicações mais recorrentes materno-infantil.

\section{Metodologia}

Trata-se de uma revisão sistemática da literatura de caráter qualitativo, relacionado a complicações por IST durante gravidez. A pesquisa trata-se do levantamento de toda bibliografia já publicada em forma de livros, revistas, publicações avulsas em imprensa escrita. De acordo com Esperón (2018), sua finalidade é colocar o pesquisador em contato direto com tudo aquilo que foi escrito sobre determinado assunto, com o objetivo de permitir ao cientista o reforço paralelo na análise de suas pesquisas ou manipulação de suas informações.

O levantamento bibliográfico foi realizado entre agosto e setembro de 2021, foi utilizada como questão norteadora, "ist mais recorrentes na gestação e suas principais complicações".

Para identificar estudos acerca do assunto realizou-se buscas nas bases de dados eletrônicas, Latino Americana (LILACS), US National Library of Medicine (PUBMED), Cumulative Index to Nursing \& Allied Health Literature (CINAHL), SciVerse Scopus (SCOPUS), Cochrane Library (COCHRANE) e Google Scholar. Com a seguinte estratégia de busca por meio do cruzamento dos descritores utilizando a lógica dos recursos booleanos no campo de busca "AND" ou "OR": (IST) AND (PREGNANCY)) OR (Infecções sexualmente transmissíveis)) AND (PREGNANCY)). A seleção dos estudos não restringiu idiomas.

Com a busca inicial foram encontrados 411 artigos que tratavam sobre a IST na gravidez, IST durante a gestação, IST mais comuns na gravidez, quais são as principais IST. Após a realização de análise do título e do resumo detalhado, foram selecionados 26 artigos que estavam relacionados com a temática da pesquisa, os quais foram lido na Integra utilizou-se 17 pesquisas para discutir os resultados, esses correspondiam com a ótica do trabalho.

Para a extração e análise dos dados incluídos e excluídos, elaborou-se um quadro de extração de dados, apêndice A. A seleção dos artigos foi norteada por critérios de inclusão, sendo: artigos cujos títulos e/ou resumos indicassem se tratar de um estudo sobre IST na gravidez sendo relativo ao conceito, à prevenção, diagnóstico e/ou tratamento, disponíveis na íntegra de forma gratuita e online, no período entre 2016 e 2021. A seleção dos estudos não restringiu idiomas. Os critérios de exclusão 
foram: textos que se tratem apenas de resumos de artigos, resenhas, comentários, trabalhos duplicados, artigos não disponibilizados para visualização completa e/ou que não atendam ao critério de inclusão.

\section{Resultados e Discussão}

Os artigos nos quais foram pesquisados e analisados que se encontram na Tabela 1, comprovam resultados obtidos através de estudos pertinentes ao tema IST: Suas Principais Complicações durante a gravidez, no período entre 2017 e 2021 . No quadro abaixo foram inseridos os dados e resultados pertinentes ao tema pesquisado, separados por autor, ano, país, objetivos, população, tamanho da amostra, método, tipo de intervenção e duração.

Tabela 1. Artigos elegíveis para o estudo.

\begin{tabular}{|c|c|c|c|}
\hline $\begin{array}{l}\text { AUTOR/ANO/PAÍS } \\
\text { DE ORIGEM }\end{array}$ & OBJETIVOS & MÉTODO & $\begin{array}{c}\text { PRINCIPAIS ACHADOS SOBRE A } \\
\text { REVISÃO }\end{array}$ \\
\hline $\begin{array}{l}\text { Trindade et al. } \\
\qquad \begin{array}{c}\text { (2021) } \\
\text { Brasil }\end{array}\end{array}$ & $\begin{array}{l}\text { Analisar o } r \\
\text { epidemiológico }\end{array}$ & $\begin{array}{l}\text { Estudo analítico com } \\
\text { abordagem quantitativa. }\end{array}$ & $\begin{array}{l}\text { O aumento progressivo de casos de HIV/aids em } \\
\text { mulheres em idade reprodutiva contribuiu para o } \\
\text { incremento nas taxas de transmissão vertical, } \\
\text { apresentando-se como importante desafio para as } \\
\text { políticas públicas de saúde. }\end{array}$ \\
\hline $\begin{array}{l}\text { Aleluia et } \\
\text { al. } \\
\text { (2021) }\end{array}$ & $\begin{array}{l}\text { Conhecer as repercussões } \\
\text { da sífilis na gestação e a } \\
\text { possibilidade de atuação da } \\
\text { enfermagem. }\end{array}$ & $\begin{array}{l}\text { Revisão integrativa } \quad \text { da } \\
\text { literatura. }\end{array}$ & $\begin{array}{l}\text { A sífilis leva a sequelas a curto e longo prazo se } \\
\text { não tiver o tratamento adequado para frear suas } \\
\text { repercussões. Para a genitora as consequências } \\
\text { vão desde físicas à sofrimento psíquico, e durante } \\
\text { a gestação, qua- } \\
\text { ndo ocorre a transmissão vertical, pode ocasionar } \\
\text { abortamento, baixo peso ao nascer, } \\
\text { prematuridade, natimorto e óbitos perinatais. }\end{array}$ \\
\hline $\begin{array}{l}\text { Mouta et al. } \\
\qquad \begin{array}{l}\text { (2018) } \\
\text { Brasil }\end{array}\end{array}$ & $\begin{array}{l}\text { Conhecer os fatores } \\
\text { relacionados ao não uso de } \\
\text { medidas preventivas das } \\
\text { infecções sexualmente } \\
\text { transmissíveis durante o } \\
\text { período gravídico. }\end{array}$ & $\begin{array}{l}\text { Estudo descritivo, } \\
\text { exploratório, com abordagem } \\
\text { qualitativa. }\end{array}$ & $\begin{array}{l}\text { Cabe ressaltar que, assim como o vírus da } \\
\text { imunodeficiência humana (HIV), a sífilis, as } \\
\text { hepatites e o HTLV (vírus linfotrópico de células } \\
\text { T humanas), existem outras IST com potenciais } \\
\text { complicações sobre a gestação e o feto, tais como } \\
\text { as infecções por Neisseria gonorrhoea e } \\
\text { Chlamydia trachomatis, que podem acarretar } \\
\text { sofrimento materno, aborto, parto prematuro, } \\
\text { morte fetal, doenças congênitas e morte do recém- } \\
\text { nascido. }\end{array}$ \\
\hline $\begin{array}{l}\text { Soares et al. } \\
\qquad \begin{array}{l}\text { (2017) } \\
\text { Brasil }\end{array}\end{array}$ & $\begin{array}{l}\text { Descrever a ocorrência de } \\
\text { sífilis gestacional e } \\
\text { congênita em Guarapuava- } \\
\text { PR, segundo } \\
\text { características maternas, } \\
\text { neonatais e evolução dos } \\
\text { casos. }\end{array}$ & $\begin{array}{l}\text { Estudo } \\
\text { retrospectivo. }\end{array}$ & $\begin{array}{l}\text { No mundo, } 1.360 .000 \text { de gestantes foram } \\
\text { infectadas, } 80 \% \text { dessas foram atendidas em } \\
\text { programas de pré-natal, } 38 \% \text { apresentaram algum } \\
\text { resultado adverso, como mortalidade fetal e } \\
\text { neonatal, prematuridade ou baixo peso ao nascer e } \\
\text { infecção congênita. }\end{array}$ \\
\hline $\begin{array}{l}\text { Horta et al. } \\
\text { (2017) } \\
\text { Brasil }\end{array}$ & $\begin{array}{l}\text { Destacar a importância do } \\
\text { envol-vimento do } \\
\text { parceiro, durante a } \\
\text { gestação, para saúde do } \\
\text { tri-nômio (gestante-bebê- } \\
\text { parceiro), enfatizando a } \\
\text { prevenção da sífilis } \\
\text { congênita na extensão da } \\
\text { assistência pré-natal do } \\
\text { parceiro. }\end{array}$ & Revisão bibliográfica. & $\begin{array}{l}\text { Dentre as complicações ocorri-das em gestantes } \\
\text { com sífilis temos o aborto espontâneo, natimorto, } \\
\text { baixo peso ao nascer, complicações precoces ou } \\
\text { tardias e prematuridade. }\end{array}$ \\
\hline Pontes et al. & $\begin{array}{l}\text { Analisamos os elementos } \\
\text { do contexto de produção e } \\
\text { o dispositivo de }\end{array}$ & Descritivo e exploratório. & $\begin{array}{l}\text { Do ponto de vista da análise do dispositivo de } \\
\text { enunciação, ficou evidente o predomínio do tema } \\
\text { da prevenção da transmissão vertical do HIV. }\end{array}$ \\
\hline
\end{tabular}




\begin{tabular}{|c|c|c|c|}
\hline $\begin{array}{l}(2020) \\
\text { Brasil }\end{array}$ & $\begin{array}{l}\text { enunciação de } 14 \text { materiais } \\
\text { educativos } \\
\text { prevenção da sobre } \\
\text { HIV/Aidis e } \\
\text { gestantes. }\end{array}$ & & $\begin{array}{l}\text { Nessa abordagem, prevalece o discurso biomédico } \\
\text { que, frequentemente, reitera a responsabilidade } \\
\text { pelo cuidado com a saúde da criança à gestante, } \\
\text { ratificando a necessidade da testagem no pré- } \\
\text { natal. }\end{array}$ \\
\hline $\begin{array}{l}\text { Dittus et al. } \\
\qquad(2020) \\
\text { Estados Unidos }\end{array}$ & $\begin{array}{l}\text { Levantamento de dados de } \\
\text { testes de DST realizados } \\
\text { em mulheres grávidas nos } \\
\text { Estados Unidos, 2011- } \\
2015 .\end{array}$ & $\begin{array}{l}\text { análises bivariadas e } \\
\text { multivariadas, examinamos } \\
\text { associações entre dados } \\
\text { demográficos, acesso a } \\
\text { cuidados de saúde e duas } \\
\text { variáveis de resultado - } \\
\text { recebimento do teste de } \\
\text { clamídia e recebimento de } \\
\text { outro teste de DST. }\end{array}$ & $\begin{array}{l}\text { Adquirir uma DST durante a gravidez é } \\
\text { especialmente problemático, pois muitas DSTs } \\
\text { podem ser assintomáticas e podem resultar em } \\
\text { uma série de complicações. } 4 \text { A transmissão } \\
\text { vertical de algumas DSTs durante a gravidez } \\
\text { pode causar sequelas significativas, como } \\
\text { resultados adversos da gravidez, baixo peso ao } \\
\text { nascer, cegueira, problemas neurológicos e de } \\
\text { desenvolvimento e natimorto. }\end{array}$ \\
\hline $\begin{array}{l}\text { Trinh et al. } \\
\qquad \text { (2019) } \\
\text { Estados Unidos }\end{array}$ & $\begin{array}{l}\text { Realizar } r \text { revisões } \\
\text { sistemáticas para localizar } \\
\text { documentos de orientação } \\
\text { que descrevem o manejo } \\
\text { da sífilis na gravidez, } \\
2003-2017 \text {. }\end{array}$ & $\begin{array}{l}\text { revisões sistemáticas e meta- } \\
\text { análises (PRISMA). }\end{array}$ & $\begin{array}{l}\text { A sífilis é transmitida por exposição sexual ou de } \\
\text { mãe para filho durante a gravidez. Quando não } \\
\text { tratada, estima-se que a sífilis materna resulte em } \\
\text { resultados adversos no parto (ABOs) em } 50-80 \% \\
\text { das gestações afetadas, dependendo do estágio da } \\
\text { sífilis na mulher. ABOs associados à sífilis são } \\
\text { frequentemente graves, com natimortos (mais } \\
\text { comumente) ou morte neonatal sendo } \\
\text { responsáveis por mais da metade dos resultados } \\
\text { ruins. Prematuridade, baixo peso ao nascer e bebês } \\
\text { com infecção congênita também são comuns se a } \\
\text { sífilis na gravidez não for tratada ou tratada } \\
\text { tardiamente. }\end{array}$ \\
\hline $\begin{array}{c}\text { Bezerra et al. } \\
\text { (2019) } \\
\text { Brasil }\end{array}$ & $\begin{array}{l}\text { Avaliar a sífilis congênita } \\
\text { como um preditor de } \\
\text { qualidade da atençõ básica } \\
\text { materno-in fatil no Brasil } \\
\text { no período de 2010-2015. }\end{array}$ & $\begin{array}{l}\text { Abordagem quantitativa para } \\
\text { realizar um estudo ecológico } \\
\text { de dados epidemiológicos } \\
\text { agregados sobre sífilis em } \\
\text { mulheres grávidas e crianças. }\end{array}$ & $\begin{array}{l}\text { A sífilis congênita é considerada um evento } \\
\text { sentinela da qualidade da assistência pré-natal } \\
\text { ( } \underline{26} \text { ). Vimos uma correlação entre a falta de } \\
\text { assistência pré-natal e as taxas de natimortos } \\
\text { devido à sífilis congênita. Também vimos uma } \\
\text { correlação entre as taxas de sífilis congênita e as } \\
\text { taxas de mortalidade infantil, aborto espontâneo e } \\
\text { natimorto. Essas correlações reforçam a hipótese } \\
\text { de que a sífilis congênita é um preditor da } \\
\text { qualidade da assistência pré-natal e revelam as } \\
\text { precárias condições de atenção à saúde materno- } \\
\text { infantil no Brasil. Os desfechos adversos podem } \\
\text { estar relacionados à falha no diagnóstico ou } \\
\text { tratamento inadequado da sífilis em gestantes, o } \\
\text { que, por sua vez, demonstra fragilidades na } \\
\text { atenção básica à saúde materno- infantil }\end{array}$ \\
\hline $\begin{array}{c}\text { Souza et al. } \\
\qquad \begin{array}{c}\text { (2019) } \\
\text { Brasil }\end{array}\end{array}$ & $\begin{array}{l}\text { Identificar a sazonalidade } \\
\text { dos casos notificados de } \\
\text { sífilis em uma região do } \\
\text { estado de São Paulo. }\end{array}$ & $\begin{array}{l}\text { Coleta de dados e análises } \\
\text { estatísticas. }\end{array}$ & $\begin{array}{l}\text { A Sífilis é uma Infecção Sexualmente } \\
\text { Transmissível (TSI) com significativa } \\
\text { importância para a saúde pública, devido ao seu } \\
\text { impacto durante a gravidez (Sífilis Gestacional - } \\
\text { GS); especialmente porque a sífilis pode afetar o } \\
\text { desenvolvimento do feto e neonatos (transmissão } \\
\text { de mãe para filho - transmissão vertical da sífilis), } \\
\text { aumentando a suscetibilidade ao aborto, parto } \\
\text { prematuro, malformações esqueléticas, meningite } \\
\text { e pneumonia. }\end{array}$ \\
\hline $\begin{array}{l}\text { Tareke et al. } \\
\text { (2019) } \\
\text { África }\end{array}$ & $\begin{array}{l}\text { Investigar a } \\
\text { soroprevalência e os } \\
\text { fatores de risco associados } \\
\text { à sífilis em gestantes } \\
\text { atendidas no pré-natal no } \\
\text { Hospital de Referência }\end{array}$ & $\begin{array}{l}\text { Estudo transversal } \\
\text { prospectivo. }\end{array}$ & $\begin{array}{l}\text { Apesar da disponibilidade de antibióticos baratos } \\
\text { e eficazes, a sífilis continua sendo uma doença } \\
\text { prevalente nos países em desenvolvimento e } \\
\text { ressurgiu como uma ameaça à saúde pública nos } \\
\text { países desenvolvidos [ } \underline{3} \text { ]. Estima-se que } 36 \\
\text { milhões de pessoas estejam infectadas com sífilis } \\
\text { em todo o mundo, com } 12 \text { milhões de novas } \\
\text { infecções relatadas a cada ano, das quais } 2 \text { milhões }\end{array}$ \\
\hline
\end{tabular}




\begin{tabular}{|c|c|c|c|}
\hline & $\begin{array}{l}\text { Felege Hiwot, noroeste da } \\
\text { Etiópia. }\end{array}$ & & $\begin{array}{l}\text { são mulheres grávidas. Mais da metade das } \\
\text { mulheres infectadas transmitem a infecção a seus } \\
\text { bebês, resultando em resultados adversos da } \\
\text { gravidez, incluindo morte fetal precoce, } \\
\text { natimorto, parto prematuro, baixo peso ao nascer, } \\
\text { morte neonatal e infecção congênita em bebês. }\end{array}$ \\
\hline $\begin{array}{l}\text { Biadgo et al. } \\
\text { (2019) } \\
\text { África }\end{array}$ & $\begin{array}{l}\text { Determinar } \\
\text { soroprevalência de } \\
\text { infecções por HIV e sífilis } \\
\text { entre mulheres grávidas na } \\
\text { clínica Gondar Family } \\
\text { Guidance Association, } \\
\text { noroeste da Etiópia. }\end{array}$ & Um estudo retrospectivo. & $\begin{array}{l}\text { As infecções sexualmente transmissíveis (IST) } \\
\text { estão entre as doenças mais disseminadas do } \\
\text { mundo, particularmente em países de baixa renda. } \\
\text { A sífilis é uma doença sistêmica causada } \\
\text { pelo Treponema pallidum. A infecção pode ser } \\
\text { classificada como congênita ou adquirida, } \\
\text { transmitida por meio de relação sexual insegura ou } \\
\text { transfusão de sangue, e é causa de resultados } \\
\text { adversos na gravidez, como perda fetal e parto } \\
\text { prematuro. Em } 2012 \text {, afetou quase um milhão de } \\
\text { mulheres grávidas; } 350.000 \text { tiveram resultados } \\
\text { adversos na gravidez em todo o mundo. }\end{array}$ \\
\hline $\begin{array}{c}\text { Botswana } \\
\text { Haiti } \\
\text { Peru } \\
\text { África do Sul e Vietnã. }\end{array}$ & $\begin{array}{l}\text { Avaliar a aceitabilidade a } \\
\text { viabilidade de triagem e } \\
\text { tratamento de CT, NG e } \\
\mathrm{TV} \text { entre mulheres } \\
\text { grávidas em } 6 \text { países de } \\
\text { baixa e média renda. }\end{array}$ & Estudos de rastreamento. & $\begin{array}{l}\text { Todos os anos, há cerca de } 349 \text { milhões de novas } \\
\text { infecções por Chlamydia } \\
\text { trachomatis (CT), Neisseria gonorrhoeae (NG) } \\
\text { e Trichomonas vaginalis (TV) em todo o } \\
\text { mundo. 1 Em mulheres grávidas, essas infecções } \\
\text { podem estar associadas a resultados adversos } \\
\text { graves no parto, incluindo trabalho de parto } \\
\text { prematuro, parto prematuro e bebês com baixo } \\
\text { peso ao nascer. 2-4 Além disso, a infecção de TC } \\
\text { ou NG materna durante o parto pode causar a } \\
\text { transmissão de TC ou NG de mãe para filho em } \\
30 \% \text { a } 70 \% \text { dos casos. 5-7A infecção neonatal por } \\
\text { TC pode causar oftalmia neonatal por clamídia e } \\
\text { pneumonia por clamídia. A infecção neonatal NG } \\
\text { pode causar oftalmia neonatal gonocócica que, se } \\
\text { não tratada, pode levar à cegueira. Finalmente, a } \\
\text { infecção materna com CT, NG ou TV pode estar } \\
\text { associada a uma maior chance de adquirir a } \\
\text { infecção pelo HIV e uma maior probabilidade de } \\
\text { transmissão vertical do HIV. }\end{array}$ \\
\hline Silva et al. (2020) & $\begin{array}{l}\text { realizar uma revisão } \\
\text { integrativa para identificar } \\
\text { na literatura científica } \\
\text { aquelas publicações que } \\
\text { apresentem o nível de } \\
\text { conhecimento de gestantes } \\
\text { sobre IST's e seus riscos na } \\
\text { gravidez. }\end{array}$ & $\begin{array}{l}\text { revisão integrativa (RI) da } \\
\text { literatura que é um método de } \\
\text { pesquisa que possibilita uma } \\
\text { avaliação crítica, a síntese e a } \\
\text { análise do nível de } \\
\text { conhecimento acerca de } \\
\text { determinado assunto, e que se } \\
\text { encontra disponível em bases } \\
\text { de dados de conhecimento } \\
\text { científico (USP, 2015). }\end{array}$ & $\begin{array}{l}\text { Gestantes que compõem os artigos pesquisados se } \\
\text { encontram em condição de vulnerabilidade sobre } \\
\text { as formas de contágio e tratamento das IST's } \\
\text { devido principalmente a apresentarem baixo nível } \\
\text { de escolaridade, dependência econômica e afetiva } \\
\text { de seus parceiros }\end{array}$ \\
\hline $\begin{array}{l}\text { Grant et al. } \\
\qquad(2020) \\
\text { Estados Unidos }\end{array}$ & $\begin{array}{l}\text { Descrever as principais } \\
\text { considerações e lacunas de } \\
\text { conhecimento sobre a } \\
\text { triagem etiológica de DST } \\
\text { durante a gravidez usando } \\
\text { as estruturas PICO e } \\
\text { GRADE. }\end{array}$ & $\begin{array}{l}\text { Revisão narrativa baseou-se } \\
\text { em pesquisas bibliográficas } \\
\text { do PubMed, na revisão das } \\
\text { diretrizes da OMS e de outras } \\
\text { agências e na opinião de } \\
\text { especialistas. }\end{array}$ & $\begin{array}{l}\text { Esta revisão examinou as lacunas de evidência em } \\
\text { torno do rastreamento etiológico de DST na } \\
\text { gravidez em países de baixa e média renda para } \\
\text { cada um dos critérios GRADE para orientar a } \\
\text { pesquisa em andamento que poderia apoiar o } \\
\text { desenvolvimento de diretrizes internacionais. }\end{array}$ \\
\hline $\begin{array}{l}\text { Teasdale et al. } \\
\qquad \text { (2018) } \\
\text { Estados Unidos }\end{array}$ & $\begin{array}{l}\text { Objetivo principal de } \\
\text { comparar a incidência } \\
\text { entre mulheres grávidas e } \\
\text { não grávidas. }\end{array}$ & $\begin{array}{l}\text { Os dados vieram do estudo } \\
\text { Métodos para Melhoria da } \\
\text { Saúde Reprodutiva na África } \\
\text { (MIRA), um ensaio clínico } \\
\text { aberto do diafragma e gel } \\
\text { lubrificante para prevenção da } \\
\text { infecção por HIV em } \\
\text { mulheres que foi aprovado por } \\
\text { conselhos de revisão } \\
\text { institucional (IRB) nos }\end{array}$ & $\begin{array}{l}\text { Embora nossa análise tenha mostrado que } \\
\text { mulheres grávidas tinham taxas de incidência de } \\
\text { DST mais altas em comparação com mulheres não } \\
\text { grávidas, especialmente de clamídia, a gravidez } \\
\text { não aumentou o risco de adquirir qualquer uma } \\
\text { das infecções em modelos ajustados. Uma análise } \\
\text { anterior desses dados, restrita apenas a mulheres } \\
\text { que tiveram uma gravidez, descobriu que } \\
\text { enquanto a atividade sexual diminuiu durante a }\end{array}$ \\
\hline
\end{tabular}




\begin{tabular}{|c|c|c|c|}
\hline & & $\begin{array}{l}\text { Estados Unidos, Sul África e } \\
\text { Zimbábue. }\end{array}$ & $\begin{array}{l}\text { gravidez, o uso de preservativo também foi menor } \\
\text { entre as mulheres grávidas. }\end{array}$ \\
\hline $\begin{array}{l}\text { Yeganeh et al. } \\
\qquad \text { (2020) } \\
\text { Los Angeles }\end{array}$ & $\begin{array}{l}\text { avaliar a aceitabilidade da } \\
\text { oferta de testagem e } \\
\text { tratamento para IST a } \\
\text { gestantes no sul do Brasil, } \\
\text { bem como avaliar as taxas } \\
\text { de prevalência e os fatores } \\
\text { de risco para IST. }\end{array}$ & $\begin{array}{l}\text { oferecemos a todas as } \\
\text { mulheres grávidas testes } \\
\text { clínicos de IST para } \\
\text { anticorpos do HIV e } \\
\text { anticorpos treponêmicos (via } \\
\text { teste de fluxo lateral de testes } \\
\text { rápidos fornecidos pelo } \\
\text { governo brasileiro) } \\
\text { para Neisseria } \\
\text { gonorrhoeae, Chlamydia } \\
\text { trachomatis e Trichomonas } \\
\text { vaginalis (via polimerase teste } \\
\text { baseado em reação em cadeia } \\
\text { fornecido por Gene Xpert, } \\
\text { Sunnyvale, CA) em 10 } \\
\text { clínicas públicas de saúde pré- } \\
\text { natal em Porto Alegre. As } \\
\text { mulheres participantes } \\
\text { responderam a uma breve } \\
\text { pesquisa por meio de um } \\
\text { instrumento de pesquisa } \\
\text { assistido por computador } \\
\text { sobre dados demográficos, } \\
\text { parcerias e comportamentos } \\
\text { sexuais. }\end{array}$ & $\begin{array}{l}\text { estudo foi limitado porque investigou apenas a } \\
\text { prevalência pontual de DSTs e não acompanhou } \\
\text { prospectivamente as mulheres durante a gravidez } \\
\text { para investigar a doença incidente }\end{array}$ \\
\hline $\begin{array}{c}\text { Kassa et al. (2018) } \\
\text { Dinamarca }\end{array}$ & $\begin{array}{l}\text { Este estudo foi realizado } \\
\text { para determinar as } \\
\text { tendências na } \\
\text { soroprevalência das quatro } \\
\text { principais infecções } \\
\text { sexualmente } \\
\text { transmissíveis (ISTs) } \\
\text { (HIV, vírus da hepatite B } \\
\text { (HBV), vírus do herpes } \\
\text { simplex tipo } 2 \text { (HSV-2) e } \\
\text { sífilis) [...] em mulheres } \\
\text { grávidas na Etiópia. }\end{array}$ & $\begin{array}{l}\text { Mulheres grávidas ( } 15-49 \\
\text { anos de idade) que estavam } \\
\text { inscritas na vigilância } \\
\text { nacional de HIV com base em } \\
\text { cuidados pré-natais foram } \\
\text { incluídas. Os rastes } \\
\text { sorológicos para HIV, HBV, } \\
\text { HSV-2 e sífilis foram feitos } \\
\text { em amostras de soro / plasma. }\end{array}$ & $\begin{array}{l}\text { Um declínio em HIV, HBV, HSV-2 e sífilis de> } \\
40 \% \text { foi observado ao longo dos anos na } \\
\text { Etiópia. No entanto, um nível intermediário de } \\
\text { endemicidade de HBV e maior prevalência de } \\
\text { HIV e HSV-2 até } 2014 \text {, sugere a necessidade de } \\
\text { fortalecer a estratégia de prevenção para DSTs. }\end{array}$ \\
\hline $\begin{array}{c}\text { Ayala et al. (2018) } \\
\text { Brasil }\end{array}$ & $\begin{array}{l}\text { Analisar os casos } \\
\text { notificados/confirmados } \\
\text { de sífilis gestacional em } \\
\text { Joinville - SC. }\end{array}$ & $\begin{array}{l}\text { Estudo descritivo, com } \\
\text { abordagem quantitativa, que } \\
\text { realizou de forma } \\
\text { retrospectiva a análise dos } \\
\text { casos notificados/confirmados } \\
\text { de gestantes com sífilis. }\end{array}$ & $\begin{array}{l}\text { mulheres jovens, sem ocupação remunerada e } \\
\text { baixa renda estão mais suscetíveis a Infecções } \\
\text { Sexualmente Transmissíveis. Outros achados } \\
\text { foram o diagnóstico tardio da sífilis entre as } \\
\text { gestantes e a condição de parceiros não tratados. }\end{array}$ \\
\hline $\begin{array}{c}\text { Moimaz et al. (2017) } \\
\text { Brasil }\end{array}$ & $\begin{array}{l}\text { Verificar os principais } \\
\text { aspectos que envolvem a } \\
\text { gestação de alto risco, } \\
\text { relatar as principais } \\
\text { alterações bucais durante a } \\
\text { gestação e salientar os } \\
\text { cuidados em saúde bucal. }\end{array}$ & $\begin{array}{l}\text { Trata-se de uma pesquisa de } \\
\text { literatura na qual foram } \\
\text { consultadas as principais } \\
\text { bases de artigos científicos das } \\
\text { Ciências da Saúde: Scielo, } \\
\text { Lilacs, Medline e BBO, } \\
\text { publicados nos últimos } 10 \\
\text { anos. }\end{array}$ & $\begin{array}{l}\text { O conhecimento de aspectos da saúde geral e } \\
\text { bucal, relacionado à gestação de alto risco e aos } \\
\text { cuidados necessários, possibilita a melhora na } \\
\text { qualidade da prestação de serviços. }\end{array}$ \\
\hline $\begin{array}{c}\text { Chaponda et al. (2021) } \\
\text { Califórnia }\end{array}$ & $\begin{array}{l}\text { Este estudo estimou a } \\
\text { prevalência de infecções } \\
\text { curáveis sexualmente } \\
\text { transmissíveis e do trato } \\
\text { reprodutivo (DSTs / RTIs) } \\
\text { entre mulheres grávidas } \\
\text { atendidas em cuidados pré- } \\
\text { natais (ANC). }\end{array}$ & $\begin{array}{l}\text { Informações } \\
\text { sociodemográficas e amostras } \\
\text { biológicas foram coletadas, e } \\
\text { a prestação de cuidados } \\
\text { baseados em gestão } \\
\text { sindrômica foi documentada. }\end{array}$ & $\begin{array}{l}\text { DSTs / RTIs curáveis eram comuns e a maioria } \\
\text { dos casos não foram detectados e } \\
\text { tratados. Abordagens alternativas são } \\
\text { urgentemente necessárias no cenário do ANC na } \\
\text { Zâmbia rural. }\end{array}$ \\
\hline $\begin{array}{c}\text { Ramos et al. (2020) } \\
\text { Brasil }\end{array}$ & $\begin{array}{l}\text { O presente artigo aborda } \\
\text { as infecções que causam } \\
\text { úlcera genital, tema que } \\
\text { compõe o Protocolo }\end{array}$ & $\begin{array}{l}\text { Foram realizadas seleção e } \\
\text { análise das evidências } \\
\text { disponíveis e discussão em } \\
\text { painel de especialistas, além }\end{array}$ & $\begin{array}{l}\text { Os aspectos clínicos das úlceras genitais são } \\
\text { bastante variados e têm baixa relação de } \\
\text { sensibilidade e especificidade com o agente } \\
\text { etiológico.O diagnóstico com base na impressão }\end{array}$ \\
\hline
\end{tabular}




\begin{tabular}{|c|c|c|c|}
\hline & $\begin{array}{l}\text { Clínico e Diretrizes } \\
\text { Terapêuticas (PCDT) para } \\
\text { Atenção Integral às } \\
\text { Pessoas com Infecções } \\
\text { Sexualmente } \\
\text { Transmissíveis (IST). }\end{array}$ & $\begin{array}{l}\text { da aprovação pela Comissão } \\
\text { Nacional de Incorporação de } \\
\text { Tecnologias no Sistema Único } \\
\text { de Saúde (Conitec). }\end{array}$ & $\begin{array}{l}\text { clínica apresentou valores preditivos positivos de } \\
30,9 \% \text { para sífilis e } 32,7 \% \text { para cancroide, sem } \\
\text { correlação clínica para casos com cancro misto no } \\
\text { Brasil. }\end{array}$ \\
\hline Goiás (2018) & $\begin{array}{l}\text { Definição de caso de } \\
\text { infecção pelo HIV em } \\
\text { indivíduos menores de } 13 \\
\text { anos de idade. }\end{array}$ & $\begin{array}{l}\text { Evidência de diagnóstico de } \\
\text { infecção pelo HIV por teste } \\
\text { sorológico (de triagem, } \\
\text { confirmatório e teste rápido) } \\
\text { ou virológico, normatizados } \\
\text { pelo Ministério da Saúde. }\end{array}$ & $\begin{array}{l}\text { Ratificamos que, toda criança de gestantes HIV } \\
\text { deve ser notificada como "Criança Exposta ao } \\
\text { HIV" e monitorada até os } 18 \text { meses de vida e caso } \\
\text { a criança seja diagnóstica com o vírus, passa a ser } \\
\text { notificada conforme o agravo. Após a implantação } \\
\text { de testes rápidos nas Estratégias de Saúda da } \\
\text { família bem como em maternidades é possível } \\
\text { identificar gestantes e tratá-la adequadamente, } \\
\text { reduzido o risco de transmissão vertical. }\end{array}$ \\
\hline
\end{tabular}

Fonte: Silva et al. (2021).

\subsection{Apuração a Respeito das IST's mais comuns durante a gravidez}

Os artigos analisados na Tabela 1 demonstram resultados correspondentes entre os estudos. Conforme os artigos analisados e selecionados constitui a respeito de tais casos correspondentes ao tema proposto. As gestantes que não fazem uso de preservativos são as que correm mais riscos de contrair alguma IST e não obtiveram as informações com um profissional qualificado da área da saúde.

Analisando as IST's recorrentes na gravidez, destacam-se o vírus da imunodeficiência humana (HIV), a sífilis, as hepatites e o HTLV (vírus linfotrópico de células T humanas), existem outras IST com potenciais complicações sobre a gestação e o feto, tais como as infecções por Neisseria gonorrhoea e Chlamydia trachomatis, que podem acarretar sofrimento materno, aborto, parto prematuro, morte fetal, doenças congênitas e morte do recém-nascido.

Para Dittus et al (2020) as infecções sexualmente transmissíveis (IST) encontram-se entre as principais causas de procura por assistência no mundo, com impactos econômicos, sociais e sanitários significativos. Afetam ambos os sexos e tornam o organismo mais vulnerável às outras doenças, além de terem relação direta com a mortalidade materna e infantil. As IST correspondem a qualquer doença que pode ser transmitida de uma pessoa para outra por meio do contato sexual, o qual pode ser oral-genital, oral-anal, relações sexuais anais, ou qualquer outro tipo de relação sexual por contato. (Dittus et al. 2018).

Conforme Silva et al (2020) Infecções Sexualmente Transmissíveis (IST) tem origem em dezenas de tipos de vírus e bactérias, sendo transmitidas, principalmente através do contato sexual, com pessoa infectada e sem o uso de preservativos. Com essa holística, há uma necessidade de não apenas as gravidas terem o cuidado de contrair ou transmitir doenças aos seus filhos e parceiros como também a sociedade em geral ter a consciência de manter o sexo seguro, pois o grande afetado é a gestante que fica vulnerável no período de gestação como o feto, colocando em risco vossas vidas.

Outro item extremamente importante ser discutido, é que no período gestacional, durante o parto ou a amamentação, a mãe também pode transmitir IST à criança (Silva et al. 2020).

É relevante o que o autor destaca, pois acredita-se que o período mais crítico é quando o bebê está no ventre, há uma mínima atenção durante e o pós parto principalmente com a amamentação, se orienta que o leite materno é o melhor a oferecer ao bebê, de fato, mais precisa elencar que o leite é o melhor quando a mãe está saudável, do contrário o leite pode ser o produto que colocará em risco a vida da criança.

Segundo Violari et al (2008) o HIV, como as outras ISTs, tem um efeito severo em mulheres grávidas. Estudos têm mostrado resultados adversos da gravidez em mulheres infectadas pelo HIV Foi relatado em um estudo que a restrição de crescimento intrauterino (RCIU) $(20,5 \%$ vs. $6,3 \%)$, parto pré-termo $(25,0 \%$ vs. $9,8 \%)$ e parto cesáreo $(45,5 \%$ vs. $29,8 \%$ ) foram significativamente mais frequentes entre mulheres com infecções por HIV não tratadas na gravidez do 
que em mulheres que receberam terapia antirretroviral altamente ativa (HAART) desde o início da gravidez Além disso, na ausência de qualquer intervenção, as taxas de transmissão do HIV de mãe para filho variam de $15 \%$ a $45 \%$. Por outro lado, crianças infectadas com HIV e não tratadas apresentam maior risco de mortalidade nos primeiros 3 meses de vida, e até $50 \%$ morrem em 2 anos.

As infecções maternas que podem afetar o feto podem ser detectadas quando existem recursos disponíveis. Em muitos casos, a primeira infecção pode ser assintomática e o diagnóstico dependerá de métodos efetivos de rastreamento. Em muitos lugares, os testes mais específicos e sensíveis não estão facilmente disponíveis. Se a infecção materna é confirmada, em geral é difícil confirmar a infecção do embrião/feto. O rastreamento preconcepção e pré-natal na mãe, ou neonatal na criança, quando está disponível, tem uma importância fundamental para o diagnóstico e tratamento precoces (Moimaz et al. 2017).

Portanto, fica claro que realizar o acompanhamento das mães durante a gravidez ou no neonatal é axial para se diagnosticar as possíveis doenças que venham surgir e realizar um tratamento eficaz a saúde da mãe e da criança, reduzindo assim, as contaminações e consequentemente os óbitos nessa faze de desenvolvimento (Horta et al. 2017).

\subsection{Constatação das intercorrências mais encontradas relacionadas à IST na gestação.}

Com a análise dos resultados encontrados pode-se perceber que em todos os estudos analisados as complicações mais recorrentes na transmissão vertical das IST são aborto, baixo peso ao nascer, cegueira, problemas neurológicos e de desenvolvimento, prematuridade, natimorto e óbitos. Sobretudo está relacionada ao não tratamento ou tratamento tardio das gestantes que apresente algum tipo de Infecção Sexualmente Transmissível (Chaponda, 2020).

Cabe ressaltar que, assim como o vírus da imunodeficiência humana (HIV), a sífilis, as hepatites e o HTLV (vírus linfotrópico de células T humanas), existem outras IST com potenciais complicações sobre a gestação e o feto, tais como as infecções por Neisseria gonorrhoea e Chlamydia trachomatis, que podem acarretar sofrimento materno, aborto, parto prematuro, morte fetal, doenças congênitas e morte do recém-nascido. (Mouta, et al 2018).

Então, adquirir uma IST durante a gravidez é especialmente problemático, pois muitas IST podem ser assintomáticas e podem resultar em uma série de complicações. A transmissão vertical de algumas IST durante a gravidez pode causar sequelas significativas, como resultados adversos da gravidez baixo peso ao nascer, cegueira, problema neurológico e de desenvolvimento e natimorto. (Dittus et al. 2020).

Para tanto, as ISTs prejudicam consideravelmente as gestante quanto a vida dos fetos, posteriormente durante o nascimento e pós nascimento, isso é bem visível, principalmente pelo $\mathrm{n}^{\mathrm{o}}$ expressivo de crianças que possuem algum tipo de deficiência em virtude das ISTs não terem sido tratadas antes da gravidez, isso leva-se a sugerir que é necessário se fazer um trabalho de orientação não apenas as grávidas e sim as pessoas a terem o sexo seguro, se sabe que já existe vários trabalhos nesse sentido nas escolas como temas transversais, mais que tal se passar no nas associações de bairros e etc, locais onde uma parte da sociedade participam, quem sabe assim se terá uma redução satisfatória quanto o índice das ISTs.

\section{Considerações Finais}

Os estudos obtidos, através dos resultados encontrados, possuem informações a respeito das IST mais diagnosticas em gestantes e seus potenciais complicações durantes a gravidez, após do nascimento do RN e em alguns casos ao longo de toda a sua vida.

A partir da realização desse estudo foi possível a análise das produções, que destacou as principais IST, sua fisiopatologia, formas de transmissão, seus efeitos adversos na gestação, e também tratamento e formas de prevenções. 
Identificou-se o quão prejudicial pode ser contrair uma IST durante a gestação, podendo ocasionar várias complicações para genitora, que vão desde físicas á sofrimento psíquico, e também ao feto quando ocorre a transmissão vertical, podendo ocasionar baixo peso ao nascer, prematuridade, cegueira, aborto espontâneo e até mesmo morte fetal.

Observaram-se casos em que o diagnóstico precoce não era possível pela falta testes rápidos na unidade de saúde em que era realizado o pré-natal, e sabemos que esse acompanhamento e essencial para o bem estar da gestante e seu bebê e que o diagnóstico precoce é muito importante para evitar possíveis complicações futuras. Sendo assim considerado as IST um sério problema de saúde pública.

Diante disso, podemos perceber a importância do profissional Enfermeiro nessas situações, principalmente na atenção básica através do pré-natal, onde é realizado o teste rápido a cada trimestre da gestação. Então durante esse período ela ao obter um diagnóstico precoce da doença, e seguidamente ser ofertado o tratamento adequado evitará complicações fatais, visto que, a paciente se encontra em uma fase muito delicada, onde deixa a mulher mais sensível durante todo o período gestacional, e se o enfermeiro poder-lhe proporcionar um acolhimento cidadão, conforme o resultado dos testes ela se sentirá firme diante da situação para fazer o seu tratamento, do contrário o psicológico dessas gestantes ficarão abalados apara enfrentar essa dificuldades. Por isso, fica claro a função do enfermeiro no acolhimento, no acompanhamento, no diagnóstico e tratamento das mães com qualquer complicação na gestação e no neonatal.

Dessa forma sugere - se a realização de novos estudos que possam discutir o conhecimento das mulheres e das gestantes sobre as complicações que as IST's podem causar na gravidez, as intervenções de enfermagem em casos de IST associada ao quadro gravídico.

\section{Agradecimentos}

Agradecemos a Deus, por ter nos guiado, dado saúde e graça, pelo Seu amor e misericórdia derramada sobre nossas vidas durante todo o período de graduação até aqui e desde sempre.

Agradecemos a nossos pais e familiares pela dedicação, amor e paciência.

A nossa orientadora, Prof. a Enf. a Ingrid da Silva Leite, por todo ensinamento e suporte, pelas correções e incentivos.

À todos, agradecemos.

\section{Referências}

Aleluia, E. S., Nascimento, L. R., Bomfim, V. V. B. S., Rodrigues, A. S., Arruda, M. D. I. S., Oliveira., M. E. W. B. S., Couto, S. I. S., Ramos, T. H. V., Daniela de Oliveira Holanda., David Matias Souza. (2021). Repercussões da sífilis na gestação: Possibilidades de atuação da enfermagem. Pesquisa, Sociedade e Desenvolvimento, 10(7), p. 2525-3409, 2021. DOI: http://dx.doi.org/10.33448/rsd-v10i7.16944

Andrade, N. C. (2021). Oliveira. Infecção por HIV em gestantes e os desafios para o cuidado pré-natal. Revista Brasileira de Enfermagem. 2021; 74 (4):e20190784. https://www.scielo.br/j/reben/a/bBbKgXFybMqFpsvm5ScBFWv/?format=pdf\&lang=pt.

Ayala, A. L. M., Biliski, M. J. B., Jasko, B. G. D. (2021). Análise da sífilis em gestantes nos anos de 2010 a 2019 em Joinville - SC. Espaço Saúde 2021;22:e762. DOI: $10.22421 / 1517-7130 /$ es.2021v22.e762

Bezerra M. L. M. B., Fernandes, F. E. C. V., Nunes, J. P. O., Baltar, S. L. S. M. A., \& Randau, K. P. (2019). Congenital Syphilis as a Measure of Maternal and Child Healthcare, Brazil. Emerging Infectious Diseases. Vol. 25(8), August 2019. DOI: 10.3201/eid2508.180298

Biadgo, B., Hassen, A., Getaneh, M., Tesfa, H., Jaleta, K. N., Eshetu, T., Kasew, D., \& Melku, M. (2018). Syphilis and human immunodeficiency virus infections among pregnant women attending antenatal care clinic of Gondar family guidance association, Northwest Ethiopia: implication for prevention of mother to child transmission. Reproductive Health (2019) 16:27. DOI:https://doi.org/10.1186/s12978-019-0691-z.

Chaponda, E. B., Bruce, J., Michelo, C., Chandramohan, D., \& Chico, R. M. (2021). Assessment of syndromic management of curable sexually transmitted and reproductive tract infections among pregnant women: an observational crosssectional study. BMC Pregnancy and Childbirth, 2021. https://bmcpregnancychildbirth.biomedcentral.com/track/pdf/10.1186/s12884-021-03573-3.pdf.

Dittus, P. J., Gift, T. L., Haderxhanai, L. T., Leichliter, J. S. (2020). Division of STD Prevention, Centers for Disease Control and Prevention, Atlanta GA, United States. Biblioteca Nacional de Medicina dos EUA no Instituto Nacional de Saúde. (2020). https://www.ncbi.nlm.nih.gov/pmc/articles/PMC7078010/.

Esperón, J. M. T. (2017). Pesquisa quantitativa na ciência da enfermagem. Escola Anna Nery. 21(1) Rio de Janeiro, Epub Feb $16,2017$. 
Goiás. (2018). Situação Epidemiológica dos casos de HIV/Aids no Estado de Goiás. Boletim Epidemiológico HIV/Aids - 2018. file:///C:/Users/INTELBRAS/Downloads/ARTIGO\%20ANY\%202\%20(1).pdf.

Grant, J. S., Chico, R. M., Lee, A. C., Low, N., Marino, A. M., Molina, R. L., Morroni, C., Masire, D. R., Stafylis, C., Tang, W., Vallely, A. J., Wynn, A., Yeganeh, N., \& Klausner, J. D. (2020). Sexually Transmitted Infections in Pregnancy: A Narrative Review of the Global Research Gaps, Challenges, and Opportunities. Sexually Transmitted Diseases, 47(12), 2020. DOI: 10.1097/OLQ.0000000000001258

Horta, H. H. L., Martins, M. F., Nonato, T. F., \& Alves, Monica. (2017). Pré - Natal do Parceiro na Prevenção da Sífilis Congênita. International Journal Of Infectious Diseases. Revista APS. n 4, p. 623 - 627, 2017. DOI: https://doi.org/10.34019/1809-8363.2017.v20.16078

Joseph O., Biodun O., \& Michael E. (2011). Resultado da gravidez entre mulheres HIV positivas recebendo HAART pré-natal versus infecção materna por HIV não tratada. Journal of College of Physicians and Surgeons Pakistan. 2011; $21: 356-359$.

Kassa, D., Gebremichael, G., Tilahun, T., Ayalkebet, A., Abrha, Y., Mesfin, G., Belay, Y., Demissie, M., Gebrexiabher, A., \& Assefa, Y. (2019). Prevalence of sexually transmitted infections (HIV, hepatitis B virus, herpes simplex virus type 2, and syphilis) in pregnant women in Ethiopia: Trends over 10 years (20052014). International Journal of Infectious Diseases, v. 79 p.50-57, 2019. https://www.ijidonline.com/article/S1201-9712(18)34588-0/fulltext.

Melo, J. (2018). Aparecida et. at. Infecções genitais e fatores de risco em gestantes atendidas em um serviço de saúde pública. Revista Espaço para a Saúde. 2018 Ago; 19(1):82-90. Disponível em: https://docs.bvsalud.org/biblioref/2018/12/967632/8-584-911-1-ed.pdf.

Moimaz, S. A. S., R, D. T., Saliba, T. A. S., \& Garbin, C. A. S. (2017). Aspectos da saúde geral e bucal de gestantes de alto risco: revisão da literatura. Journal of the Health Sciences Institute. 35(3), P. 223 a 230, 2017.https://repositorio.unip.br/wpcontent/uploads/2020/12/V35n32017p223a2 30.pdf.

Mouta, R. J. O., de Oliveira, C. L., Medina, E. T., Prata, J. A., Correia, L. M., \& da Mota, C. P. (2018). Fatores Relacionados Ao Não Uso De Medidas Preventivas Das Infecções Sexualmente Transmissíveis Durante A Gestação. Revista Baiana De Enfermagem, 32. DOI: https://doi.org/10.18471/rbe.v32.26104.

Pontes, B. S., Santos, A. K., \& Monteiro, S. (2020). Produção de discursos sobre a prevenção do HIV/Aids e da sífilis para gestantes em materiais educativos elaborados por instituições brasileiras (1995 - 2017). Interface (Botucatu). 2020. DOI:https://doi.org/10.1590/Interface.190559

Ramos, M. C., Sardinha, J. C., Alencar, H. D. R., Aragón, M. G., \& Lannoy, L. H. (2021). Protocolo Brasileiro para Infecções sexualmente transmissíveis 2020: infecções que causam úlcera genital. Epidemioliologia Serviços Saúde, Brasília, 30(1):e2020663, 2021. https://www.scielo.br/j/ress/a/t5cFGq4BcJW 3b4NvDq9y7dz/?format=pdf\&lang=pt.

Shannon, C. L., Bristow, C., Hoff, N., Wynn, A., Nguyen, M., Marino, A. M., Cabeza, J., Rimoin, A., \& Klausner, J. D. (2018). Short Report: Acceptability and Feasibility of Rapid Chlamydial, Gonococcal, and Trichomonal Screening and Treatment in Pregnant Women in Six Low-to-Middle Income Countries. Published in final edited form as: Sexually Transmitted Diseases publishes. 2018 October; 45 (10): 673-676. DOI:10.1097/OLQ.0000000000000832.

Silva, F. C., Medeiros, H. A. O., Anjos, T. F., \& Viana, T. C T. (2020). Infecções Sexualmente Transmissíveis Em Gestantes: Uma Revisão Integrativa. Brazilian Journal of Surgery and Clinical Research - $\quad$ BJSCR. 33(2), pp. 98-103 (Dez 2020 - Fev 2021). https://www.mastereditora.com.br/periodico/20210108_095204.pdf.

Soares, L. G., Zarpellon, B., Soares, L. G., Baratieri, T., Lentsck, M. H., \& Mazza, V. A. (2017). Sífilis gestacional e congênita: características maternas, neonatais e desfecho dos casos. Revista Brasileira de Saúde Materno Infantil, Recife, 17(4): 791-799 out. / dez., 2017. https://www.scielo.br/j/rbsmi/a/M97FZ bnrgbCxk7RjbwSJSv/?format=pdf\&lang=pt.

Souza, J. M., Giuffrida, R., Ramos, A. P. M., Morceli, G., Coelho, C. H., \& Rodrigues, M. V. P. (2019). Mother-to-child transmission and gestational syphilis: Spatial-temporal epidemiology and demographics in a Brazilian region. (2019). PLOS Neglected Tropical Diseases. DOI:https://doi.org/10.1371/journal. pntd.0007122

Tareke, K., Munshea, A., \& Nibret, E. (2019). Seroprevalence of syphilis and its risk factors among pregnant women attending antenatal care at Felege Hiwot Referral Hospital, Bahir Dar, northwest Ethiopia: a cross-sectional study. BMC Res Notes.12:69 DOI:https://doi.org/10.1186/s13104-019-4106-6.

Teasdale, C. A., Abrams, E. J., Chiasson, M. A., Justman, J., Blanchard, K., \& Jones, H. E. (2018). Incidence of sexually transmitted infections during pregnancy. (2018). PLoS ONE 13(5): e 0197696. DOI:https:// doi.org/10.1371/journal.pone.0197696.

Trindade, L. N. M., Nogueira, L. M. V., Rodrigues, I. L. A., Ferreira, A. M. R., Corrêa, G. M., \& Andrade, N. C. O. (2021). Infecção por HIV em gestantes e os desafios para o cuidado pré-natal. Revista Brasileira de Enfermagem. 2021;74 (4):e20190784. DOI: https://doi.org/10.1590/0034-7167-2019-0784

Trinh, T., Leal, A. F., Mello, M. B., Taylor, M. M., Barrow, R., Wi, T. E., \& Kamb, M. L. (2019). Syphilis management in pregnancy: a review of guideline recommendations from countries around the world. Sexual and Reproductive Health Matters, 27(1), p. 69-82, 2019. DOI: 10.1080/26410397.2019.1691897.

Violari, A., Cotton, M. F., Gibb, D. M., Babiker, A. G., Steyn, J., Madhi, S. A., Philippe, P. J., \& McIntyre, J. A. (2008). Early antiretroviral therapy and mortality among HIV-infected infants. O New England Journal of Medicine. 2008. Nov 20; 359(21):2233-44. DOI: 10.1056/NEJMoa0800971.

Yeganeh, N., Kreitchmann, R., Leng, M., Saines, K. N., Gorbach, P. M., \& Klausner, J. (2021). High Prevalence of Sexually Transmitted Infections in Pregnant Women Living in Southern Brazil. Sexually Transmitted Diseases 48(2), February 2021. DOI: 10.1097/OLQ.0000000000001276 